\title{
INTRODUCTION TO THE SPECIAL ISSUE ON CAUSAL DISCOVERY
}

\author{
Shohei Shimizu* and Jun-ichiro Hirayama**
}

\begin{abstract}
Many empirical sciences, including the social sciences and life sciences, aim to study causal relationships. Researchers in these fields need computational methods for analyzing observed data and identifying causal structures among a set of variables. Such computational methods enable researchers to draw conclusions on the basis of both their assumptions and the observed data. Moreover, these methods are useful for developing hypotheses on causal relations, designing future observational studies, and planning future experimental studies that can potentially provide stronger evidence of estimated causal relations.

The objective of this special issue is to present an up-to-date overview of causal discovery methods, which have witnessed rapid advancements in recent years. The chief editor and guest editors invited the following three survey papers on various hot topics related to causal discovery:
\end{abstract}

1. Introduction to nested Markov models by Ilya Shpitser (University of Southampton, UK), Robin Evans (University of Oxford, UK), Thomas Richardson (University of Washington, USA) and James Robins (Harvard University, USA)

\section{Learning causal structure from multiple datasets with similar variable} sets by Robert E. Tillman (Blander Technologies, Chicago, IL, United States) and Frederick Eberhardt (California Institute of Technology, USA)

\section{LiNGAM: Non-Gaussian methods for estimating causal structures by Shohei Shimizu (Osaka University, Japan)}

The guest editor Shimizu was assigned to the first two papers, while the guest editor Hirayama was assigned to the third one. All three papers were reviewed by two reviewers so as to elicit suggestions for possible improvements in their presentation.

In the first paper, Shpitser, Evans, Richardson, and Robins examine a recent causal model called the nested Markov model, which considers latent confounding variables and models a marginal distribution of observed variables using both, conditional independence constraints on observed variables, and more general constraints induced in cases where latent confounding variables exist. This approach enables the identification of more causal relations than that using only conditional independence constraints.

In the second paper, Tillman and Eberhardt discuss recent nonlinear nonparametric methods based on the conditional independence of observed variables for estimat-

* The Institute of Scientific and Industrial Research, Osaka University, Mihogaoka 8-1, Ibaraki, Osaka 567-0047, Japan. E-mail: sshimizu@ar.sanken.osaka-u.ac.jp

** Cognitive Mechanisms Laboratories, Advanced Telecommunications Research Institute International (ATR), Hikaridai 2-2-2, Seika-cho, Soraku-gun, Kyoto 619-0288, Japan. E-mail: hirayama@atr.jp 
ing causal relationships from multiple datasets, possibly with different variable sets. Considering latent confounders is necessary to effectively use information contained in multiple datasets. This approach can provide better estimation performance than previous approaches based on a single independent and identically distributed (i.i.d) dataset.

In the third paper, Shimizu reviews a non-Gaussian framework of structural equation models with parametric functional forms. This framework utilizes the nonGaussianity of observed data for model identification and enables the estimation of more causal relations than conventional Gaussian methods in many cases, including latent confounding variable cases.

The three invited papers cover a wide range of causal discovery approaches and emphasize the importance of considering latent confounding variables. We believe that these papers provide a useful snapshot of current trends in causal discovery, and they would find many practical applications.

We are greatly indebted to our authors and guest editorial board members. We are also grateful to the Behaviormetrika editorial board, in particular, the chief editor Maomi Ueno, for giving us the opportunity to present this special issue.

Guest editorial board:

-Jun-ichiro Hirayama (ATR, Japan), Guest editor

-Antti Hyttinen (University of Helsinki, Finland)

-Shohei Shimizu (Osaka University, Japan), Guest editor

-Ricardo Silva (University College London, UK)

-Maomi Ueno (University of Electro-Communications, Japan), Chief editor

-Michio Yamamoto (Kyoto University, Japan)

-Kun Zhang (Max Planck Institute for Biological Cybernetics, Germany) 